\title{
Ajuste de ecuaciones alométricas para estimar biomasa aérea en Pinus oocarpa y Quercus resinosa en Guerrero, México
}

\section{Fitting allometric equations to estimate aboveground biomass for Pinus oocarpa and Quercus resinosa at Guerrero, Mexico}

\author{
José Navarro-Martínez', Flaviano Godínez-Jaimes²*, Miguel Ángel López-López ${ }^{3}$, José Luis Rosas-Acevedo ${ }^{4}$, \\ Ana Laura Juárez-López y Maximino Reyes-Umaña ${ }^{4}$
}

\begin{abstract}
1 Universidad Autónoma de Guerrero. Centro de Ciencias de Desarrollo Regional. Doctorado en Ciencias Ambientales. Acapulco, Guerrero, México.

2 Universidad Autónoma de Guerrero. Facultad de Matemáticas. Chilpancingo, Guerrero, México.

3 Colegio de Postgraduados. Posgrado en Ciencias Forestales. Texcoco, Edo. de México, México.

4 Universidad Autónoma de Guerrero. Centro de

Ciencias de Desarrollo Regional. Acapulco, Guerrero, México
\end{abstract}

\section{RESUMEN}

Los árboles capturan y almacenan grandes cantidades de carbono en su biomasa e impactan positivamente en la mitigación de los gases de efecto invernadero (GEI). Hasta esta fecha, no existen ecuaciones alométricas que estimen la biomasa de árboles del estado de Guerrero, México. El presente trabajo tuvo como objetivo generar ecuaciones para estimar la biomasa aérea de árboles de Pinus oocarpa Schiede y Quercus resinosa Liebmann. Se realizó un muestreo destructivo de 33 árboles para la primera especie y 19 para la segunda, distribuidos en el bosque templado de la microcuenca del río Hueyapa, Guerrero, México. La biomasa de los componentes del árbol se estimó con base en el peso seco de 6 a 14 muestras por componente arbóreo, según el tamaño del árbol. Se ajustaron modelos potenciales en su forma lineal y con transformación Box-Cox. El modelo que mejor estimó la biomasa aérea en $P$. oocarpa fue el potencial y para $Q$. resinosa fue el que se obtuvo con la transformación Box-Cox, usando logaritmo del diámetro normal $\left(\ln D_{i}\right)$ y la variable combinada transformada $\left(\mathrm{D}_{i}{ }^{2} H_{i}\right)^{\prime}$ como variable independiente. Las ecuaciones alométricas generadas rellenan el vacío existente para la estimación de la biomasa aérea de estas especies forestales en el estado de Guerrero.

PALABRAS CLAVE: criterio de información de Akaike; ecuación alométrica; Río Hueyapa; transformación Box-Cox.

\section{ABSTRACT}

Trees sequester and store large amounts of carbon within their biomass, thus helping to mitigate the effects of the greenhouse gasses (GHG). To date, no allometric equations exist to estimate biomass of the forest tree species from Guerrero, Mexico. That is why the aim of the present study was to generate equations to estimate aboveground biomass for Pinus oocarpa Schiede and Quercus resinosa Liebmann. Databases consisted of 33 and 19 sample trees for the pine and oak species, respectively, all of them distributed in temperate forest within Hueyapa river micro-watershed, Guerrero, Mexico. The biomass was estimated based on the dry weight of 6 to 14 samples per tree component, according to tree size. Power models were adjusted in their linear form and with Box-Cox transformation. The model that best estimated the aboveground biomass in P. oocarpa was the power function and for Q. resinosa it was the one obtained with the BoxCox transformation, using the logarithm of the diameter at breast height $\left(\ln D_{i}\right)$ and the transformed combined variable $\left(\mathrm{D}_{i}{ }^{2} H_{i}\right)^{\prime}$ as the independent one, respectively. The allometric equations generated fill the existing vacuum for the estimating the aboveground biomass of these forest species in Guerrero state.

KEYWORDS: Akaike information criteria AIC; allometric equation; Hueyapa River; Box-Cox transformation. 


\section{INTRODUCCIÓN}

La determinación del potencial de captura y almacén de carbono por los árboles y los bosques en respuesta al cambio climático global y al aumento de las concentraciones de carbono en la atmósfera es de suma importancia en la actualidad (Ordóñez, Rivera, Tapia y Ahedo, 2015).

Las ecuaciones alométricas son utilizadas para estimar la biomasa de los árboles con datos provenientes de inventarios forestales que permiten evaluar la estructura y condición de un bosque, cuantificar la productividad, determinar el reservorio y tasa de captura de carbono y, así, valorar el impacto del ecosistema forestal en la mitigación de los gases de efecto invernadero (GEI) (Návar 2009a; Méndez-González, Luckie-Navarrete, Capó-Arteaga y Nájera-Luna, 2011; Vashum y Jayakumar 2012). Este método requiere de la medición de variables dasométricas provenientes de procesos destructivos del árbol y de la determinación de la biomasa aérea por componente estructural (Hernández-Ramos et al., 2017).

Los modelos alométricos, en su mayoría, incluyen como única variable explicativa al diámetro normal (D) del árbol (Aguirre-Calderón y Jiménez-Pérez, 2011; Baishya, 2011); sin embargo, al agregar al modelo la densidad específica, la altura total del árbol $(\mathrm{H})$ o el factor de forma, mejora la capacidad predictiva de los mismos (Návar, 2010; Solano, Vega, Eras y Cueva, 2014; Chave et al., 2015; Soriano-Luna, Ángeles-Pérez, Martínez-Trinidad, Plascencia-Escalante y Razo-Zárate, 2015; Wang, Zhang y Feng, 2018).

Para estimar la biomasa, regularmente se establecen a priori modelos lineales o no lineales de tipo potencial que usan a $\mathrm{D}$ o $D^{2} \mathrm{H}$ como variable independiente (Návar 2009a, Wang et al., 2018). Otros autores proponen un conjunto de modelos y eligen el mejor usando los estadísticos: coeficiente de determinación ajustado, error estándar de los residuos y coeficiente de variación (NávarCháidez, 2010; Domínguez-Cabrera, Aguirre-Calderón, Jiménez-Pérez, Rodríguez-Laguna y Díaz-Balderas, 2009; Méndez-González et al., 2011). Un enfoque más consiste en proponer modelos que incluyan un mayor número de variables independientes, de las cuales se seleccionan las más importantes con los procedimientos backward o forward. Estos procedimientos hacen la selección utilizando como criterio el P-valor de cada variable, el coeficiente de determinación o el estadístico Cp de Mallows (HernándezRamos et al., 2017). Hay que resaltar que, recientemente, el mejor modelo se elige usando el criterio de información de Akaike (AIC, por sus siglas en inglés), que pertenece a la familia de estadísticos de bondad de ajuste penalizados para modelos estimados por máxima verosimilitud (Solano et al., 2014; Chave et al., 2015; Montoya et al., 2018).

Un problema común en los modelos de regresión lineal es el de la colinealidad que ocurre cuando las variables independientes están correlacionadas o, en un sentido más amplio, cuando la matriz diseño está mal condicionada, lo que puede producir estimaciones grandes, de signo contrario y/o varianzas estimadas grandes (Belsley, Kuh y Welsh, 1980). Por otro lado, estos modelos se han ajustado simultáneamente con ecuaciones aparentemente no relacionadas (Návar 2009b; Hernández-Ramos et al., 2017). Un enfoque diferente para el ajuste de modelos alométricos es el método de momentos generalizado, apropiado cuando el tamaño de muestra es pequeño y existen problemas de heterocedasticidad (Wang et al., 2018).

En una revisión de literatura sobre ecuaciones alométricas generadas para ecosistemas boscosos, selvas y matorral desértico de México, publicada en revistas especializadas y tesis para 478 especies de plantas, no se hallaron ecuaciones de biomasa para los bosques ubicados en el estado de Guerrero (Rojas-García, De Jong, MartínezZurimendi y Paz-Pellat, 2015).

Lo anterior justifica el ajuste de ecuaciones alométricas para áreas geográficas específicas, especies y/o grupos de ellas de interés local (Návar 2009b, Solano et al., 2014), puesto que las características genéticas y condiciones edáficas, climáticas, métodos de manejo del bosque, disponibilidad de nutrientes y otros factores del sitio influyen en el desarrollo del árbol (Vieilledent et al., 2012). 


\section{OBJetivos}

Generar ecuaciones alométricas para estimar la biomasa aérea total y determinar la distribución de biomasa por componente estructural, para árboles de Pinus oocarpa Schiede y Quercus resinosa Liebmann, en el bosque templado de la microcuenca del río Hueyapa, Guerrero, México.

\section{MATERIALES Y MÉTODOS}

\section{Área de estudio}

El área de estudio (Fig. 1) corresponde a la microcuenca del río Hueyapa, situada entre las coordenadas geográficas $17^{\circ}$ $10^{\prime} 33^{\prime \prime}$ y $17^{\circ} 17^{\prime} 44^{\prime \prime}$ de latitud norte, $99^{\circ} 26^{\prime} 27^{\prime \prime}$ y $99^{\circ} 31^{\prime}$ 12 " de longitud oeste. Pertenece a la subcuenca río Omitlán (RH20Eb), cuenca río Papagayo (RH20E) de la Región Hidrológica No. 20 Costa Chica de Guerrero (RH20). La microcuenca se circunscribe a los municipios de Juan R. Escudero y Chilpancingo de los Bravo en la zona centro del estado de Guerrero, México. La precipitación anual fluctúa entre $1300 \mathrm{~mm}$ y $2000 \mathrm{~mm}$, con temperatura media anual de $24.0^{\circ} \mathrm{C}$ y clima cálido subhúmedo con lluvias en verano. Se asienta sobre roca ígnea intrusiva y predomina el suelo Leptosol mólico (Instituto Nacional de Estadística y Geografía [Inegi], 2010).

La microcuenca cubre un área de 7488.5 ha, con presencia de selva mediana subcaducifolia y bosque templado. La superficie de bosque templado de la microcuenca es de 2761.9 ha, de las cuales 1601.8 ha están cubiertas por bosque de pino-encino y encino-pino, y 1160.1 ha por bosque de encino; ambos ecosistemas son aledaños y se distribuyen altitudinalmente entre $600 \mathrm{~m} \mathrm{y}$ $1613 \mathrm{~m} \mathrm{snm}$.

El bosque templado presenta una densidad arbórea promedio de $397.6 \mathrm{ha}^{-1} \pm 25.2 \mathrm{ha}^{-1} \mathrm{y}$ un área basal promedio de $22.88 \mathrm{~m}^{2} \mathrm{ha}^{-1} \pm 0.92 \mathrm{~m}^{2} \mathrm{ha}^{-1}$. Las especies arbóreas predominantes son Pinus oocarpa Schiede; seis especies del género Quercus: Q. resinosa Liebm., Q. crispifolia Trel., Q. subespatulata Trel., Q. conspersa Benth., Q. elliptica Née y Q. planipocula Trel., así como árboles de otras latifoliadas en menor proporción.

\section{Método}

La muestra representativa de árboles para el ajuste de las ecuaciones alométricas fue de 33 individuos de $P$. oocarpa con diámetros entre $10 \mathrm{~cm}$ y $80 \mathrm{~cm}$ y 19 árboles de $Q$. resinosa con diámetros entre $15 \mathrm{~cm}$ y $60 \mathrm{~cm}$, distribuidos en el bosque templado de la microcuenca.

Al inicio del periodo de lluvias de 2017, 52\% de los árboles de $P$. oocarpa y $53 \%$ de los de Q. resinosa fueron derribados por vientos fuertes (Tabla 1). Estos eran los de mayor dimensión, poseían follaje íntegro, eran sanos y sin deformaciones, con la estructura físico-mecánica sin deterioro aparente. Los árboles restantes, con características apropiadas se derribaron exprofeso y todos los productos maderables generados fueron aprovechados por personas de la comunidad agraria Dos Caminos y Anexos.

Se realizaron mediciones del diámetro normal y altura total a cada árbol de la muestra. La separación y pesaje de los componentes arbóreos (fuste, ramas y follaje) se llevó a cabo en la fase de campo. El peso verde de cada componente se obtuvo con el uso de una báscula colgante marca Rhino modelo BAC-500 con capacidad máxima de $500 \mathrm{~kg}$ y precisión de $0.1 \mathrm{~kg}$. De cada componente del árbol se recolectaron y pesaron entre 6 y 14 muestras verdes (según el tamaño del árbol), que fueron trasladadas al laboratorio de Biomonitoreo y Control Biológico del Centro de Ciencias de Desarrollo Regional de la Universidad Autónoma de Guerrero, para el secado en estufa a $70^{\circ} \mathrm{C}$ hasta obtener peso constante (peso seco de la muestra) como lo establecen Soriano-Luna et al. (2015).

El peso a $70{ }^{\circ} \mathrm{C}$ por componente arbóreo se midió utilizando la metodología establecida por Medrano-Meraz, López-López, De Jong, Ángeles-Pérez y Cruz-Cobos (2017). La biomasa aérea total $\left(B_{i}\right)$, fue la suma de la biomasa de todos los componentes arbóreos aéreos. El compartimento fuste para $P$. oocarpa se midió desde la base del árbol hasta el ápice del mismo, y para Q. resinosa., el fuste se identificó siguiendo la rama principal de mayor grosor hasta su ápice. 


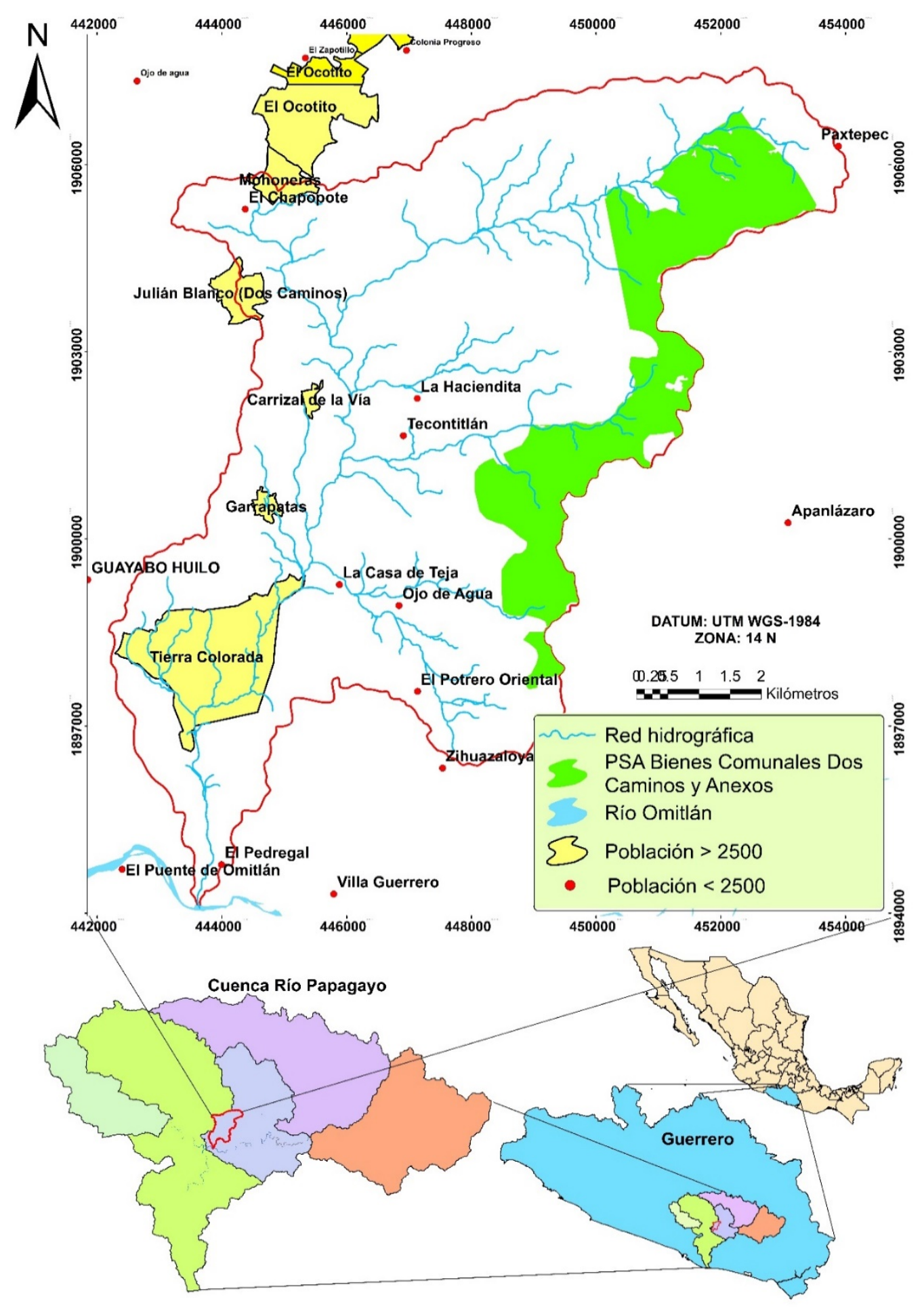

FIGURA 1. Área de estudio. 


\section{Análisis exploratorio de la muestra}

Las principales variables dasométricas medidas para los árboles muestra se registran en la tabla 2.

Tanto para $P$. oocarpa como para Q. resinosa, la relación entre $B$ y $D$, así como entre $B$ y $H$ es de tipo curvilínea. $A l$ usar el logaritmo natural o transformación de tipo Box-Cox de $B$ y graficar contra $D$, contra $H$ o contra los valores transformados de $D$ y $H$, se observó un patrón lineal. Lo anterior justifica el uso de estas transformaciones en el modelo con mejor ajuste, al mismo tiempo que permite cumplir los supuestos del modelo de regresión lineal.

\section{Ajuste de modelos}

$\mathrm{La}$ variable de respuesta fue $\mathrm{B}$ y las variables independientes: $\mathrm{D}, \mathrm{H}$ y $\mathrm{D}^{2} \mathrm{H}$ utilizando los modelos de regresión de la tabla 3.

TABLA 1. Distribución de la muestra y proporción de árboles derribados por vientos.

\begin{tabular}{llllllllllllllllll}
\hline & \multicolumn{11}{c}{ Categoría diamétrica $(\mathrm{cm})$} \\
\hline & $\mathrm{n}$ & 10 & 15 & 20 & 25 & 30 & 35 & 40 & 45 & 50 & 55 & 60 & 65 & 70 & 75 & 80 \\
\hline P. oocarpa & 33 & 1 & 1 & 1 & 2 & 2 & 4 & 4 & 2 & 4 & 2 & 3 & 2 & 2 & 1 & 2 \\
DV (P. oocarpa) & 17 & & & & & & 1 & 1 & 1 & 4 & 2 & 2 & 1 & 2 & 1 & 2 \\
Q. resinosa & 19 & & 2 & 1 & 3 & 1 & 5 & 2 & 1 & 1 & 1 & 2 & & & & \\
DV (Q. resinosa) & 10 & & & & & & 4 & 2 & 1 & 1 & 1 & 1 & & & & \\
\hline
\end{tabular}

DV: derribados por el viento, n: tamaño de muestra

TABLA 2. Estadísticas dasométricas de árboles muestra de P. oocarpa y Q. resinosa.

\begin{tabular}{cccccc}
\hline Especie & Variable & Mínimo & Media & Máximo & D. E. \\
\hline \multirow{3}{*}{ P. oocarpa } & $\mathrm{D}$ & 12.50 & 46.79 & 82.00 & 17.99 \\
& $\mathrm{H}$ & 7.15 & 21.68 & 29.10 & 4.60 \\
& $\mathrm{~B}$ & 36.91 & 1607.32 & 5856.51 & 1337.80 \\
\hline \multirow{3}{*}{ Q. resinosa } & $\mathrm{D}$ & 14.10 & 35.25 & 57.70 & 13.05 \\
& $\mathrm{H}$ & 10.40 & 16.01 & 22.80 & 3.43 \\
& $\mathrm{~B}$ & 64.31 & 997.51 & 3037.63 & 851.79 \\
\hline
\end{tabular}

D: diámetro normal (cm), H: altura total (m), B: Biomasa aérea total (kg), D. E.: desviación estándar (kg).

TABLA 3. Modelos de regresión lineal para estimar la biomasa aérea de P. oocarpa y Q. resinosa.

\begin{tabular}{|c|c|}
\hline $\ln B_{i}=\beta_{0}+\beta_{1} \ln D_{i}+\varepsilon_{i}$ & 1 \\
\hline $\ln B_{i}=\beta_{0}+\beta_{1} \ln \left(D_{i}{ }^{2} * H_{i}\right)+\varepsilon_{i}$ & 2 \\
\hline $\mathrm{B}_{i}^{\prime}=\beta_{0}+\beta_{1} \mathrm{D}_{i}{ }^{\prime}+\varepsilon_{i}$ & 3 \\
\hline $\mathrm{B}_{i}^{\prime}=\beta_{0}+\beta_{1}\left(\mathrm{D}_{i}^{2} * H_{i}\right)^{\prime}+\varepsilon_{i}$ & 4 \\
\hline $\mathrm{B}_{i}^{*}=\beta_{0}+\beta_{1} D_{i}+\beta_{2} H_{i}+\beta_{3} D_{i} H_{i}+\beta_{4} D_{i}^{2}+\beta_{5} H_{i}^{2}+\beta_{6} D_{i}^{2} H_{i}^{2}+\varepsilon_{i}$ & 5 \\
\hline
\end{tabular}


El modelo supone $\varepsilon_{i} \sim N\left(0, \sigma^{2}\right) ; i=1, \ldots, n$, es decir, que los errores tienen distribución normal, son independientes y tienen varianza constante. El ajuste del modelo de regresión se realizó con el paquete estadístico $\mathrm{R}$ (R Core Team, 2016).

Las transformaciones de las variables $B, D$ y $D^{2} H$ usadas en los modelos [3, 4 y 5$]$ se identificaron con la transformación tipo Box-Cox (Ec. 6). Estas son necesarias cuando no se cumple alguno de los supuestos de normalidad o de varianza constante, porque identifican las funciones de las variables que permiten el cumplimiento de los supuestos del modelo de regresión. La transformación tipo Box-Cox se define por:

$$
y^{\lambda}=\left\{\begin{array}{cc}
\frac{y^{\lambda}-1}{\lambda}, & \text { si } \lambda \neq 0 \\
\log (\lambda), & \text { si } \lambda=0
\end{array}\right.
$$

El parámetro $\lambda$ es el valor que maximiza la verosimilitud de los modelos estimados. Esta función fue implementada en la función powerTransform del paquete car (Fox y Weisberg, 2011) y se usó de dos formas: 1) de manera conjunta las variables $B$ y $D$ [modelo 3] y $B$ y $D^{2} H$ [modelo 4], que una vez transformadas se denotaron por $B^{\prime}$ у $D^{\prime}$ у $B^{\prime}$ у $\left(D^{2} H\right)^{\prime}$, respectivamente; 2) únicamente la variable dependiente $B_{i}$ y esta se denotó por $B_{i}^{*}$ [modelo 5].

El cumplimiento de la distribución normal de los residuos se evaluó con la prueba de Shapiro-Wilk (shapiro.test) del paquete nortest (Gross y Ligges, 2015) y la homogeneidad de varianza con la prueba de varianza no constante (ncv'Test) del paquete car (Fox y Weisberg, 2011) implementadas en R.

\section{Evaluación del modelo}

El AIC fue definido por la ecuación 7.

$$
A I C_{j}=-2 \ln \left[L\left(\hat{\theta}_{j}\right)\right]+2 p_{j}
$$

donde $L\left(\hat{\theta}_{j}\right)$ es la verosimilitud máxima estimada bajo el modelo $M_{j}$ que tiene un vector de parámetros $\theta_{j}$ de dimensión $p_{j}$ (Fox, 2016).
La colinealidad fue evaluada con el número de condición escalado de la matriz diseño (Belsley, Kuh y Welsh, 1980), definido por la ecuación 8.

$$
\eta_{x}=\sqrt{\lambda^{*}{ }_{1} / \lambda^{*} p+1}
$$

donde $\lambda^{*}{ }_{1}$ y $\lambda^{*}{ }_{p+1}$ son el máximo y el mínimo de los valores propios de $X^{T} X$ después de ser escalada. Por su intensidad, la colinealidad se clasifica en nula $\left(\eta_{x}<10\right)$, moderada $\left(10 \leq \eta_{x}<30\right)$ y severa $\left(\eta_{x} \geq 30\right)$.

El mejor modelo se identificó como aquel que tiene el menor valor de la raíz de la media de la suma de los cuadrados de los residuos (RMSCR) y la mayor correlación entre los valores de biomasa observada y estimada $\left(r^{2}\right)$, que mide la capacidad predictiva de los modelos ajustados. La RMSCR penaliza la varianza dando más peso a los residuos con mayores valores absolutos, respecto a los residuos con valores absolutos más pequeños, calculado con la ecuación 9, citado por Wang et al. (2018) como raíz del cuadrado medio del error.

$$
\operatorname{RMSCR}=\sqrt{\frac{1}{n} \sum_{i=1}^{n}\left(B_{i}-\hat{B}_{i}\right)^{2}}
$$

donde $n$ es el número de observaciones de la muestra, $B_{i}$ es la biomasa aérea total observada y $\hat{B}_{i}$ es la biomasa aérea total estimada y el residuo es la diferencia, $B_{i}-\hat{B}_{i}$.

Debido a que se ajustaron modelos tanto con las variables originales como con dos versiones de transformaciones de $B_{i}$, para determinar $\hat{B}_{i}$ se usó la función inversa de la transformación correspondiente para volver a los valores originales.

\section{Resultados}

\section{Modelación de la biomasa aérea total en $P$. oocarpa y $Q$. resinosa}

Los modelos ajustados para $P$. oocarpa y sus estadísticos de bondad de ajuste se observan en la tabla 4 y los de Q. resinosa en la tabla 5; todos cumplieron los supuestos del modelo de regresión. 
TABLA 4. Modelos ajustados para estimar la biomasa aérea total de $P$. oocarpa.

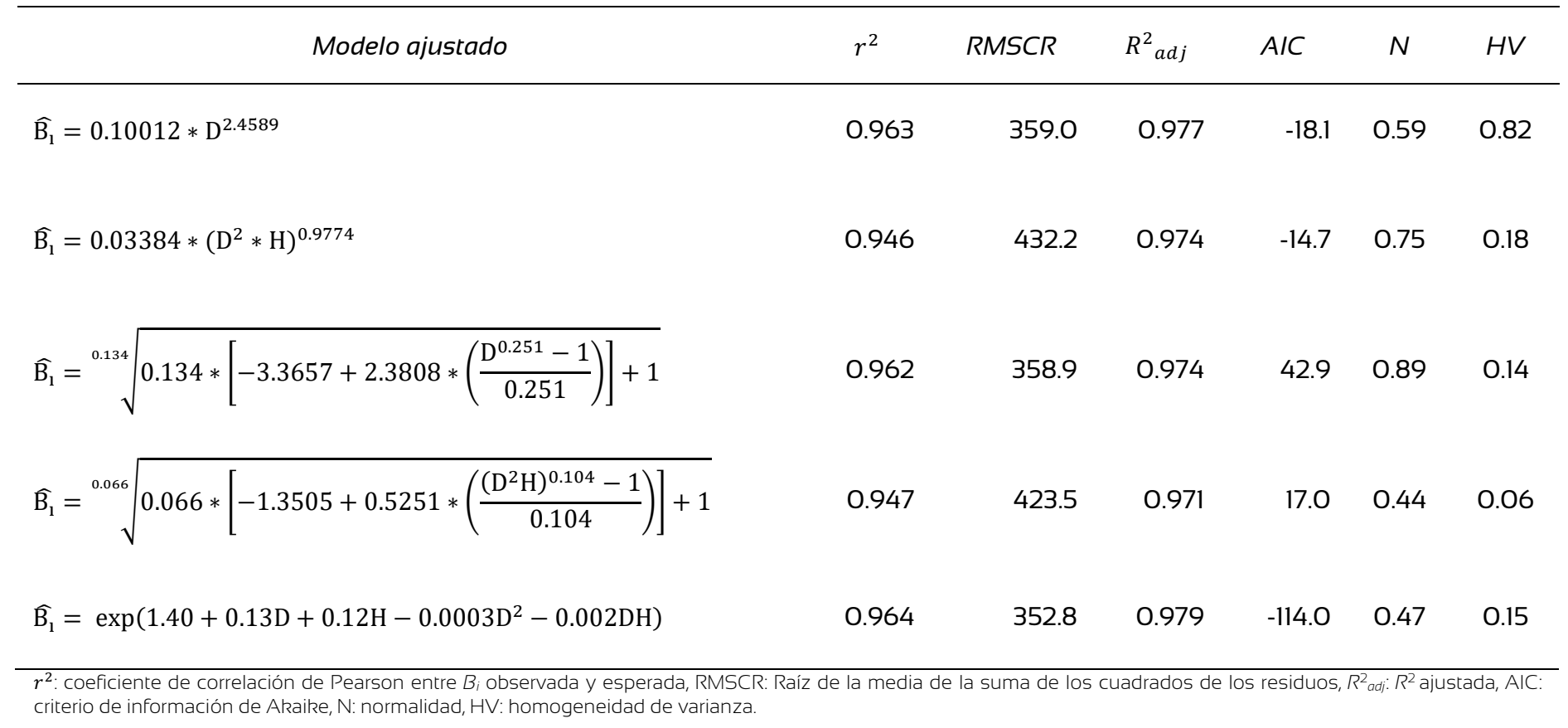

TABLA 5. Modelos ajustados para estimar la biomasa aérea total de Q. resinosa.

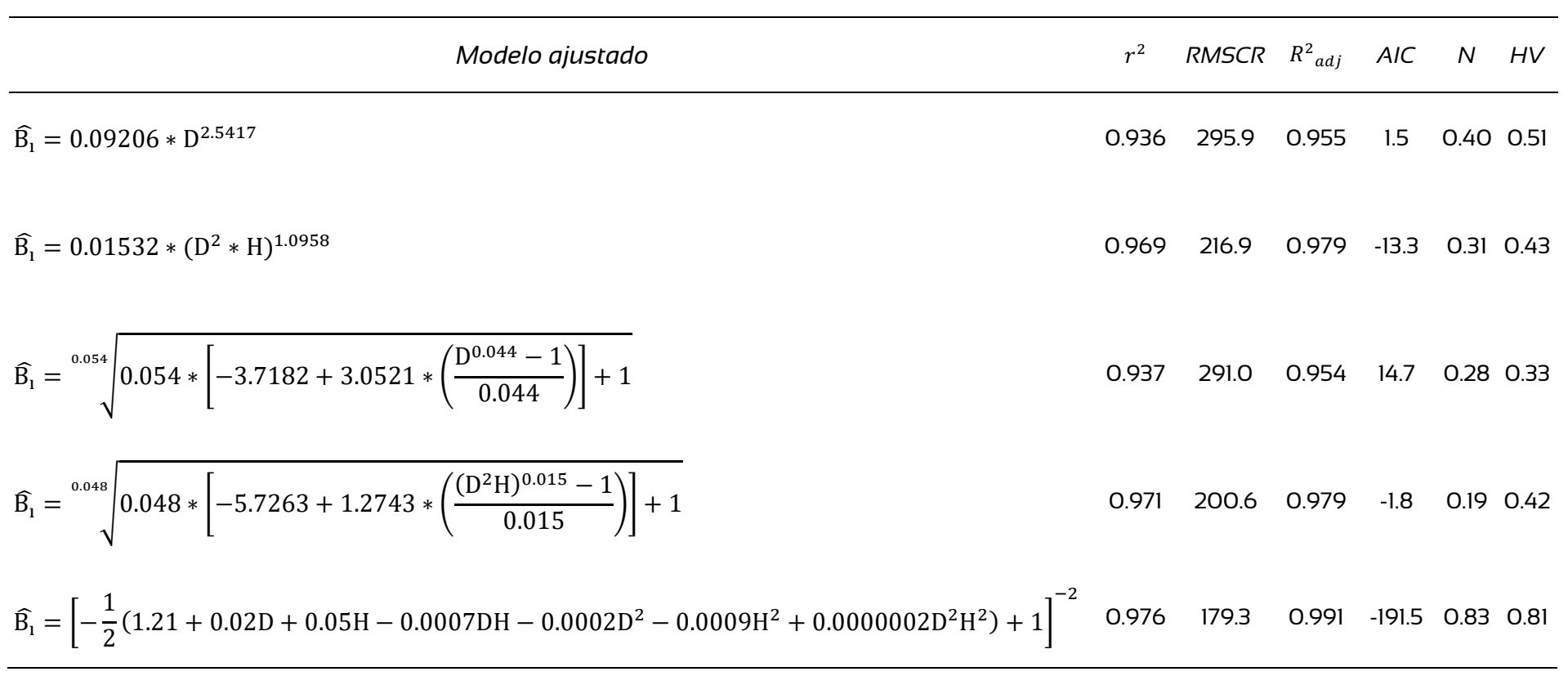


El modelo 5, generado para $P$. occarpa con transformación Box-Cox de $B_{i}{ }^{*}$ como $\ln \left(B_{i}\right)$ (Tabla 4 ) y para $Q$. resinosa con transformación

$$
B_{i}^{*}=\left[\frac{B_{i}^{*-0.05}-1}{-0.05}\right]
$$

(Tabla 5), presentó los menores valores de RMSCR y de AIC, ambos con excelente capacidad predictiva. Sin embargo, estos mostraron problemas severos de colinealidad, $\left(\eta_{x}=60\right.$ y $\eta_{x}=279$, respectivamente), por lo que no fueron elegidos como los mejores en cada caso.

Los modelos 1 y 3 de la tabla 4 presentaron valores similares de RMSCR (359.0 y 358.9) y capacidad predictiva (0.963 y 0.962); sin embargo, por el principio de parsimonia, el modelo 1 es el mejor para estimar la biomasa aérea en árboles de $P$. oocarpa.

Desde el punto de vista estrictamente estadístico, el modelo 4, ajustado para Q. Resinosa (Tabla 5), se eligió como el mejor porque explica $97.9 \%$ de la variación de $B_{i}{ }^{\prime}$, tuvo un AIC de -1.8 y capacidad predictiva de 0.971; además presentó el valor más bajo de RMSCR (200.6). El modelo 4 explicó $2.4 \%$ más de la variación de $B_{i}{ }^{\prime} \mathrm{y}$ tuvo una capacidad predictiva $3.5 \%$ superior al modelo 1 ; sin embargo, el modelo 1, que utiliza únicamente el diámetro normal como variable independiente y por la practicidad en la medición de esta variable en campo, también puede ser utilizado para la estimación de la biomasa aérea en árboles de esta especie distribuida en el bosque templado de la microcuenca del río Hueyapa.

La capacidad predictiva de los modelos elegidos como los mejores para ambas especies se observan gráficamente en la figura 2.

La distribución promedio de biomasa aérea por componente en árboles de $P$. occarpa fue de $64.7 \%$ en fuste, $32.6 \%$ en ramas, $2.0 \%$ en follaje y $0.7 \%$ en conos. La biomasa aérea promedio distribuida en árboles de Q. resinosa fue de $52.1 \%, 46.4 \%$ y $1.5 \%$ en fuste, ramas y follaje, respectivamente.

\section{DISCUSIÓN}

La corrección de la falta de normalidad y homogeneidad de varianzas de los residuos en los modelos ajustados para la estimación de la biomasa aérea mediante el uso de transformaciones de tipo Box-Cox, es tan efectiva como el uso del logaritmo para hacer lineal la función potencial (Méndez-González et al., 2011; Hernández-Ramos et al., 2017) o bien, del método de momentos generalizado (Wang et al., 2018).

El modelo alométrico ajustado por Ayala-López, De Jong y Ramírez-Maldonado (2001) para estimar la biomasa aérea en $P$. oocarpa en bosques de Chiapas, México, que usa como variables al logaritmo natural tanto para la biomasa aérea como del diámetro normal tuvo una $\mathrm{R}_{a d j}^{2}$ de 0.93 , valor similar al obtenido por Návar (2009a) para bosques del noreste de México, mientras que el modelo ajustado en este estudio tuvo un $R^{2}$ adj de 0.977 (Tabla 4), por lo que presenta una mayor explicación de la varianza de la biomasa aérea de esta especie.

González (2008) ajustó un modelo para estimar la biomasa total en $P$. occarpa que incluye al diámetro normal y su cuadrado con $R^{2}$ adj de 0.95 , valor superado por el mejor modelo ajustado (1) en este estudio, que incluyó únicamente al diámetro normal como variable independiente (Tabla 4) y que también supera la precisión de la predicción en 3.3\% respecto al modelo ajustado por Návar-Cháidez (2010).

La transformación Box-Cox usada en el ajuste del modelo elegido (4) para Q. resinosa con 19 árboles y el método de momentos generalizado utilizado por Wang et al. (2018) con 32 árboles de Q. aliena Bl. var. acuteserrata en bosques de China, tuvieron $\mathrm{R}^{2}$ adj similares de 0.98 , con el uso de las mismas variables independientes. 

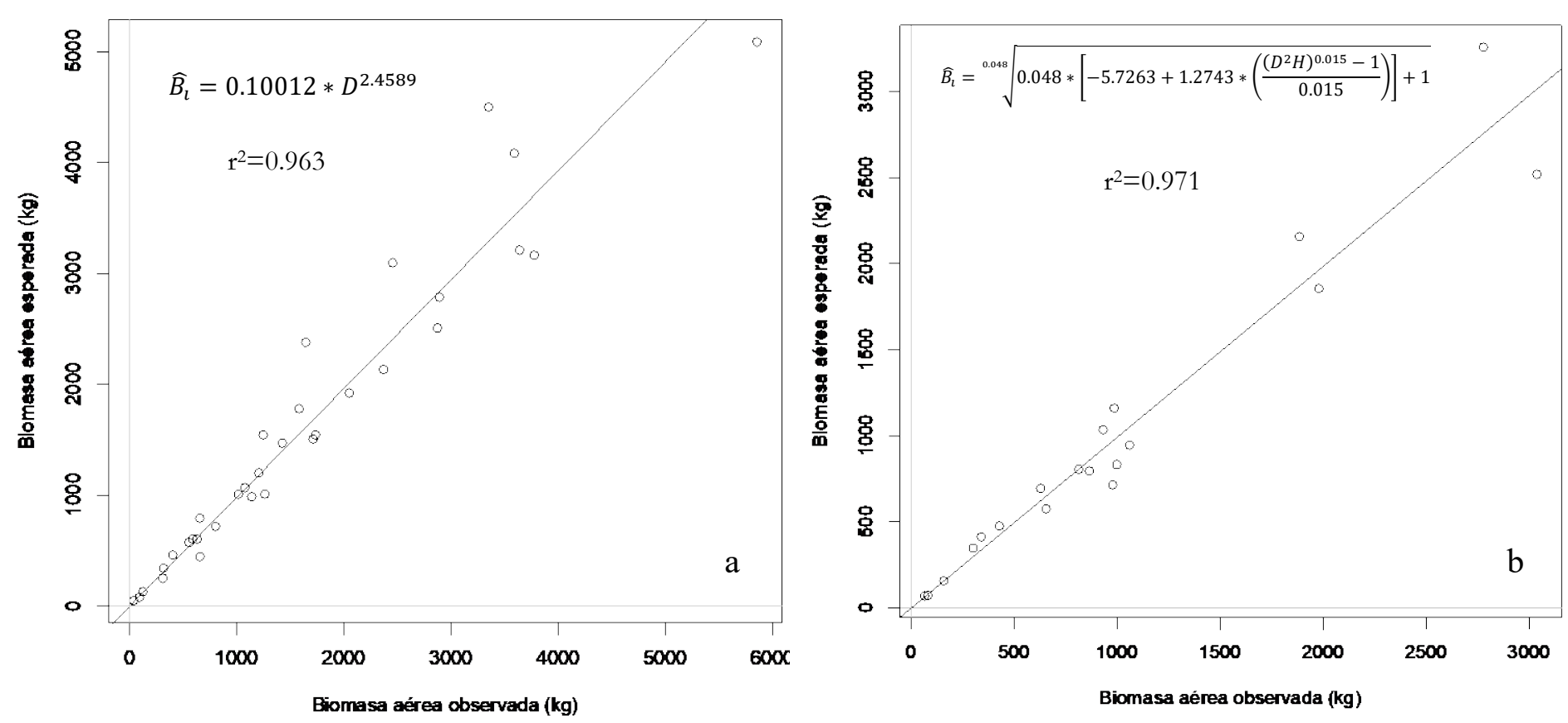

FIGURA 2. Biomasa aérea observada y esperada de $P$. oocarpa (a) y $Q$. resinosa (b).

El modelo alométrico función potencial ajustado por Chave et al. (2015) con una base de datos de 1194 árboles de regiones tropicales, que incluye el logaritmo natural de biomasa aérea y como variables independientes a la densidad básica de la madera y $D^{2} H$ tuvo un AIC de 3130, por lo que los modelos ajustados en este estudio, tanto para $P$. oocarpa como para Q. resinosa, superan este valor de bondad de ajuste con un AIC de -18.1 y -1.8 , respectivamente.

Gómez-Díaz, Etchevers-Barra, Monterrosos-Rivas, Campo-Álvarez y Tinoco-Rueda (2011) ajustaron la función potencial para estimar biomasa en Q. magnoliaefolia Née distribuida en la reserva de la biosfera Sierra de Huautla, Morelos, México, usando como variable independiente al diámetro normal, y obtuvieron un $\mathrm{R}^{2}$ adj $\mathrm{de}$ 0.98. Del mismo modo, Ruíz-Aquino et al. (2014) ajustaron la ecuación potencial con la variable combinada como independiente para estimar la biomasa aérea total en árboles de Q. laurina y Q. crassifolia en bosques de Oaxaca, México y obtuvieron un $R^{2}$ adj de 0.99 y 0.98 respectivamente, valor similar $\left(R_{a d j}^{2}=0.977\right)$ al obtenido con el mejor modelo ajustado para Q. resinosa en este trabajo (Tabla 5).
En la mayoría de los artículos publicados no se da evidencia del cumplimiento de los supuestos del modelo de regresión ajustado (normalidad, independencia y homogeneidad de varianzas), mientras que los modelos con mejor ajuste en este trabajo cumplieron con los tres supuestos, sin necesidad de utilizar la metodología de ecuaciones aparentemente no relacionadas (HernándezRamos et al., 2017) ni el método de momentos generalizado (Wang et al., 2018).

A pesar de utilizar una muestra relativamente pequeña en el ajuste de modelos para Q. resinosa $(\mathrm{n}=19)$, tanto el logaritmo natural como la transformación Box-Cox mejoran el problema de heterogeneidad de varianzas, tal como lo hace el método de momentos generalizado utilizado por Wang et al. (2018).

Por otro lado, el porcentaje de biomasa en el fuste de $P$. oocarpa fue menor al registrado para los bosques de Honduras por Ramos y Ferreira (2001) de $71.65 \%$ para fuste sin corteza y $28.35 \%$ para corteza, ramas y acículas. Para la misma especie, Alberto y Elvir (2008) observaron $71 \%$ de la biomasa concentrada en el fuste. González (2008) determinó los porcentajes de biomasa aérea para 
regeneración de $P$. oocarpa en bosques naturales en el norte de Chiapas, México de $84.8 \%, 11.2 \%$ y $4.0 \%$ para fuste, ramas y follaje, respectivamente.

En una plantación de seis años de edad de P. greggii, Pacheco et al. (2007) observaron una distribución de biomasa de $51.0 \%$ en fuste, $25.0 \%$ en ramas y $24.0 \%$ en follaje. Álvarez, Balboa, Merino y Rodríguez (2005) registraron porcentajes de biomasa aérea para $P$. pinaster en madera, corteza y copa de $68.0 \%, 11.0 \%$ y $21.0 \%$, respectivamente. Al mismo tiempo, estos autores concluyeron que, considerando un posible aprovechamiento forestal en los bosques de $P$. pinaster, los restos arbóreos esparcidos en el suelo y la reintegración de biomasa al suelo, representaría más de $21.0 \%$. Por otro lado, los resultados del presente estudio indican que, después de un aprovechamiento comercial de P. oocarpa, se reintegraría al suelo más de $35.0 \%$, lo que representa la biomasa de las ramas y follaje, debido a una gran proporción de biomasa en estos componentes.

Las copas extensas frecuentemente se producen cuando la densidad de arbolado es baja y el espacio para el crecimiento de las mismas es amplio, a este respecto, Hébert et al. (2016) encontraron que las menores densidades de plantación probadas generaron árboles de Pinus banksiana Lamb. con ramas de mayor diámetro y mayor proporción de copa viva. La competencia por la luz entre árboles vecinos explica la existencia de una mayor variación de la biomasa de ramas y de follaje entre árboles, incluso de la misma especie, como lo establece Návar (2009a), quien concluyó que los árboles que crecen con una fuerte competencia y logran una posición dominante, registran regularmente biomasa en ramas y follaje bajos comparado con árboles que crecen en espacio abierto que desarrollan copas grandes y acumulan mayor biomasa en este componente arbóreo que en el fuste.

Gómez-Díaz et al. (2011), para Q. magnoliaefolia, hallaron porcentajes de biomasa aérea de $62.5 \%$ para fuste, $27.8 \%$ para ramas y $9.6 \%$ para el follaje. En comparación con los valores encontrados en la presente investigación, la biomasa de fuste estimada por ellos es $10.4 \%$ mayor, igualmente, la biomasa de follaje encontrada por ellos es $8.1 \%$ mayor, pero la biomasa de ramas que ellos registraron fue $18.6 \%$ menor que la aquí calculada para Q. resinosa.

En un muestreo realizado en bosques de la comunidad de Ixtlán de Juárez, Oaxaca, México, Ruíz-Aquino et al. (2014) concluyeron que la biomasa de $Q$. laurina se distribuye en $82.9 \%, 14.9 \%$ y $2.2 \%$ y en $Q$. crassifolia en $82.3 \%, 16.0 \%$ y $1.7 \%$ en fuste, ramas y follaje, en el mismo orden; coincidiendo con el presente estudio en el porcentaje de biomasa en follaje en $Q$. resinosa, pero es menor en casi tres veces la biomasa en ramas, concentrando la mayor parte en el fuste, caso contrario a lo encontrado en los bosques de $Q$. resinosa de la microcuenca del río Hueyapa, Guerrero, México.

Con la finalidad de realizar una estimación precisa del reservorio de biomasa y carbono en la vegetación arbórea de la microcuenca del río Hueyapa, es preponderante generar ecuaciones alométricas para las otras cinco especies del género Quercus o bien, una ecuación para el género Quercus spp., así como para el grupo de otras latifoliadas.

\section{CONCLUSIONES}

El uso del logaritmo en la función potencial al igual que las transformaciones Box-Cox, pueden utilizarse con éxito para el cumplimiento de los supuestos del modelo de regresión. Se llena el vacío que había sobre modelos alométricos para la estimación de biomasa en dos especies forestales maderables en el estado de Guerrero. El modelo potencial 1 con el diámetro normal como variable independiente fue el de mejor ajuste para estimar la biomasa aérea en $P$. oocarpa; mientras que para $Q$. resinosa fue el modelo 4 ajustado con la transformación Box-Cox y la variable combinada como independiente.

Los modelos ajustados para ambas especies facilitarán la estimación del reservorio de carbono en el bosque templado de la microcuenca del río Hueyapa con datos provenientes de inventarios forestales. Sin embargo, es necesario incrementar la muestra de individuos de Quercus, con la finalidad de generar una ecuación genérica para el área de estudio. 


\section{RECONOCIMIENTOS}

Al Consejo Nacional de Ciencia y Tecnología (Conacyt) por el financiamiento del proyecto fondo sectorial de investigación ambiental Conacyt-Semarnat clave 278639, a las autoridades ejidales y municipales de la comunidad agraria Dos Caminos y Anexos y al grupo de trabajo de campo. A la Unidad de Educación Media Superior Tecnológica Agropecuaria y Ciencias del Mar (UEMSTAyCM) por el otorgamiento de la Beca-Comisión al primer autor para realizar sus estudios de doctorado. Así mismo, se agradece a los dos revisores anónimos que pulieron la calidad de este artículo.

\section{REFERENCIAS}

Aguirre-Calderón, O. A. \& Jiménez-Pérez, J. (2011). Evaluación del contenido de carbono en bosques del sur de Nuevo León. Revista Mexicana de Ciencias Forestales, 2(6), 73-84.

Alberto, D. M. \& Elvir, J. A. (2008). Acumulación y fijación de carbono en biomasa aérea de Pinus oocarpa en bosques naturales en Honduras. INIA. Investigaciones Agrarias: Sistemas y Recursos Forestales, 17(1), 67-78. Recuperado de http://www.inia.es/gcontrec/pub/067-078-

Acumulacion_1208252685250.pdf

Álvarez G., J. G., Balboa M., M. A., Merino, A., \& Rodríguez S., R. (2005). Estimación de la biomasa arbórea de Eucalyptus globulus y Pinus pinaster en Galicia. Recursos Rurais, 1(1), 21-30.

Ayala-López, R. S., De Jong, B. H. J., \& Ramírez-Maldonado, H. (2001). Ecuaciones para estimar biomasa en la meseta central de Chiapas. Revista Chapingo Serie Ciencias Forestales y del Ambiente, 7(2),153-157.

Baishya, S. B. (2011). Estimation of tree biomass, carbon pool and net primary production of an old-growth Pinus kesiya Royle ex. Gordon forest in orth-eastern India. Annals of Forest Science, 68(4), 727-736. doi:10.1007/s13595-011-0089-8

Belsley, D. A., Kuh, E., \& Welsh, R. (1980). Regression Diagnostics: Identifying Influential Data and Source of Collinearity. New York: John Wiley \& Sons.

Chave, J., Réjou-Méchain, M., Búrquez, A., Chidumayo, E., Colgan, M. S., Delitti, W. BC, Vieillendent, G. (2015). Improved allometric models to estimate the aboveground biomass of tropical trees. Global Change Biology, 20(10), 3177-3190. doi:10.1111/gcb.12629

Domínguez-Cabrera, G., Aguirre-Calderón, O. A., Jiménez-Pérez, J., Rodríguez-Laguna, R., \& Díaz-Balderas, J. A. (2009). Biomasa aérea y factores de expansión de especies arbóreas en bosques del sur de Nuevo León. Revista Chapingo Serie Ciencias Forestales y del Ambiente, 15(1), 59-64.

Fox, J. (2016). Applied regression analysis and generalized linear models (3a ed.) p. 674-677. London, UK: SAGE Publications, Inc.

Fox, J. \& S. Weisberg. (2011). An $\{\mathrm{R}\}$ Companion to Applied Regression (2a ed.) Thousand Oaks CA: Sage.

Gómez-Díaz, J. D., Etchevers-Barra, J. D., Monterrosos-Rivas, A. I., Campo-Álvarez, J., \& Tinoco-Rueda, J. A. (2011). Ecuaciones alométricas para estimar biomasa y carbono en Quercus magnoliaefolia. Revista Chapingo Serie Ciencias Forestales y del Ambiente, 17(2), 261-272. doi: 10.5154/r.rchscfa.2010.11.117

González, Z. M. (2008). Estimación de la biomasa aérea y captura de carbono en regeneración natural de Pinus maximinoi H.E. Moore, Pinus oocarpa var. ochoterenai Mtz. y Quercus sp. en el norte del Estado de Chiapas, México. Tesis de maestría. CATIE, Turrialba, Costa Rica. 97 p.

Gross, J., \& U. Ligges. (2015). Nortest: Tests for normality. R package versión 1.0-4. Recuperado de https://CRAN.Rproject.org $/$ package $=$ nortest

Hébert, F., Krause, C., Plourde, P., Achim, A., Prégent, G., \& Ménétrier, J. (2016). Effect of tree spacing on tree level volume growth, morphology, and wood properties in a 25-year-old Pinus banksiana plantation in the boreal forest of Quebec. Forests, 2016(7), 1-16. doi: 10.3390/f7110276

Hernández-Ramos, J., De los Santos-Posadas, H. M., Valdez-Lazalde, J. R., Tamarit-Urias, J. C., Ángeles Pérez, G., Hernández-Ramos, ... \& Carrero, O. (2017). Biomasa aérea y factores de expansión en plantaciones forestales comerciales de Eucalyptus urophylla S. T. Blake. Agrociencia, 51(8), 921-938. Recuperado de http://www.colpos.mx/agrocien/Bimestral/2017/ nov-dic/art8.pdf

Instituto Nacional de Estadística y Geografía [Inegi] (2010). Documento técnico descriptivo de la Red Hidrográfica escala 1:50000 edición 2.0. Aguascalientes, México. 106 p.

Medrano-Meraz, F., López-López, M. A., De Jong, B. H. J., ÁngelesPérez, G., \& Cruz-Cobos, F. (2017). Ecuaciones alométricas preliminares para la estimación de biomasa subterránea en Pinus patula. En F. Paz \& R. Torres (Eds.), PMC. Estado actual del conocimiento del ciclo del carbono y sus interacciones en México (pp. 431437). Síntesis 2016. Serie Síntesis Nacionales. Texcoco, Estado de México, México: Programa Mexicano del Carbono-Instituto Politécnico Nacional- Centro de Investigación y Asistencia en Tecnología y Diseño del Estado de Jalisco.

Méndez-González, J., Luckie-Navarrete, S. L., Capó-Arteaga, M. A., \& Nájera-Luna, J. A. (2011). Ecuaciones alométricas y estimación de incremento en biomasa aérea y carbono en una plantación mixta 
de Pinus devoniana Lindl y P. pseudostrobus Lindl., en Guanajuato, México. Agrociencia, 45(4), 479-491. Recuperado de http://www.scielo.org.mx/pdf/agro/v45n4/ v45n4a7.pdf

Montoya J., J. C., Méndez G., J., Sosa D., L., Ruíz G., C. G., Zermeño G., A., Nájera L., J. A., Velázquez R., A. S. (2018). Ecuaciones de biomasa aérea y volumen para Pinus halapensis Mill., en Coahuila, México. Madera y Bosques, 24(Núm. esp.), e2401880. doi: $10.21829 /$ myb.2018.2401880

Návar, J. (2009a). Allometric equations for tree species and carbon stocks for forests of northwestern México. Forest Ecology and Management, 257(2), 427-434. doi: 10.1016/j.foreco.2008.09.028

Návar, J. (2009b). Biomass component equations for Latin American species and groups of species. Annals of Forest Science, 66(2), 208. doi: 10.1051 / forest/2009001

Návar, J. (2010). Measurement and assessment methods of forest aboveground biomass: A literature review and the challenges ahead. En Momba, M. \& Bux, F. (Eds.). Biomass (pp. 27-64). Rijeka, Croatia: Sciyo. doi: 10.5772/9768

Návar-Cháidez, J. J. (2010). Biomass allometry for tree species of northwestern Mexico. Tropical and Subtropical Agroecosystems, 12(3), 507-519. Recuperado de http://www.revista.ccba.uady.mx/ojs/index.php/TSA/article/v iew/391

Ordóñez, D. J. A. B., Rivera, V. R., Tapia, M. M. E., \& Ahedo, H. L. R. (2015). Contenido y captura potencial de carbono en la biomasa forestal de San Pedro Jacuaro, Michoacán. Revista Mexicana de Ciencias Forestales, 6(32), 7-16. doi: 10.29298/rmcf.v6i32.95

Pacheco, E. F. C., Aldrete, A., Gómez, G. A., Fierros, G. A. M., Cetina A. V. M., \& Vaquera, H. H. (2007). Almacenamiento de carbono en la biomasa aérea de una plantación joven de Pinus greggii Engelm. Revista Fitotecnia Mexicana, 30(3), 251-254. Recuperado de https://www.revistafitotecniamexicana.org/documentos/303/5a.pdf

R Core Team. (2016). R: A language and environment for statistical computing. Vienna, Austria: R Foundation for Statistical Computing.

Ramos, V. S. \& Ferreira, O. W. (2001). Determinación de la cantidad y composición química de la biomasa aérea y subterránea del Pinus oocarpa. Tatascán, 13(2), 3-17.

Rojas-García, F., De Jong, B. H. J., Martínez-Zurimendi, P., \& PazPellat, F. (2015). Database of 478 allometric equations to estimate biomass for Mexican trees and forest. Annals of Forest Science, 72(6), 835-864. doi: 10.1007/s13595-015-0456-y

Ruíz-Aquino, F., Valdez-Hernández, J. I., Manzano-Méndez, F., Rodríguez-Ortiz, G., Romero-Manzanares, A., \& Fuentes-López,
M. E. (2014). Ecuaciones de biomasa aérea para Quercus laurina y Q. crassifolia en Oaxaca. Madera y Bosques, 20(2), 33-48. doi: 10.21829/myb.2014.202162

Solano, D., Vega, C., Eras, V. H., \& Cueva, K. (2014). Generación de modelos alométricos para determinar biomasa aérea a nivel de especies, mediante el método destructivo de baja intensidad para el estrato de bosque seco pluviestacional del Ecuador. Revista CEDAMAZ, 4(1), 32-44. Recuperado de https://unl.edu.ec/sites/default/files/investigacion/revistas/20 14-12-1/art_4.pdf

Soriano-Luna, M. A., Ángeles-Pérez, G., Martínez-Trinidad, T., Plascencia-Escalante, F., \& Razo-Zárate, R. (2015). Estimación de biomasa aérea por componente estructural en Zacualtipán, Hidalgo, México. Agrociencia, 49(4), 423-438

Vashum, K. T. \& Jayakumar, S. (2012). Methods to estimate aboveground biomass and carbon stock in natural forest: A review. Journal Ecosystem \& Ecography, 2:116. doi: 10.4172/21577625.1000116

Vieilledent, G., Vaudry, R., Andriamanohisoa, S. F. D., Rokotonarivo, O. S., Randrianasolo, H. Z., Razafindrabe, H. N., ...Rasamoelina, M. (2012). A universal approach to estimate biomass and carbon stock in tropical forest using generic allometric models. Ecological Applications, 22(2), 572-583. doi: 10.1890/11-0039.1

Wang, J., Zhang, L., \& Feng, Z. (2018). Allometric equations for the aboveground biomass of five tree species in China using the generalized method of moments. The Forestry Chronicle, 94(3), 214 220. doi: 10.5558/tfc2018-034.

Manuscrito recibido el 8 de mayo de 2019

Aceptado el 9 de octubre de 2019

Publicado el 31 de enero de 2020

Este documento se debe citar como:

Navarro-Martínez., J, Godínez-Jaimes, F., López-López, M. A., Rosas-Acevedo, J. L., Juárez-López, A. L., \& Reyes-Umaña, M. (2020). Ajuste de ecuaciones alométricas para estimar biomasa aérea en Pinus oocarpa y Quercus resinosa en Guerrero, México. Madera y Bosques, 26(1), e2611964. doi: 10.21829/myb.2020.2611964

Madera y Bosques por Instituto de Ecología, A.C. se distribuye bajo una Licencia Creative Commons Atribución-NoComercialCompartirlgual 4.0 Internacional. 